\title{
Detailed experimental results for laser acceleration staging
}

\author{
W. D. Kimura,* L. P. Campbell, C.E. Dilley, S. C. Gottschalk, and D. C. Quimby \\ STI Optronics, Inc., Bellevue, Washington 98004
}

A. van Steenbergen, M. Babzien, I. Ben-Zvi, J. C. Gallardo, K. P. Kusche, I. V. Pogorelsky,

J. Skaritka, and V. Yakimenko

Brookhaven National Laboratory, Upton, New York 11973

D. B. Cline, P. He, and Y. Liu

University of California, Los Angeles, Los Angeles, California 90095

L. C. Steinhauer

University of Washington, Redmond Plasma Physics Laboratory, Redmond, Washington 98052

R. H. Pantell

Stanford University, Stanford, California 94305

(Received 28 August 2001; published 26 October 2001)

\begin{abstract}
Detailed experimental results of staging two laser-driven, relativistic electron accelerators are presented. During the experiment called STELLA (staged electron laser acceleration), an inverse free-electron laser (IFEL) is used to modulate the electron energy, thereby, causing $\sim 3 \mathrm{fs}$ microbunches to form separated by the laser wavelength at $10.6 \mu \mathrm{m}$ (equivalent to a $35 \mathrm{fs}$ period). A second IFEL accelerates the electrons depending upon the phase of the microbunches entering the second IFEL with respect to the laser beam driving the second IFEL. The data presented includes electron energy spectra as a function of the phase delay and laser power driving the first IFEL. Also shown is a comparison with the computer model, which includes space charge and misalignment effects.
\end{abstract}

DOI: 10.1103/PhysRevSTAB.4.101301

PACS numbers: 41.75.Jv, 41.60.Cr

\section{INTRODUCTION}

The future of elementary particle research at the energy frontier requires the development of electron accelerators [1] able to obtain $>10 \mathrm{TeV}$ energies, which are much higher than currently possible with microwave-based accelerators. Present microwave accelerators are reaching their maximum acceleration gradient limits $(\sim 100 \mathrm{MeV} /$ $\mathrm{m})$. Assuming a gradient of $100 \mathrm{MeV} / \mathrm{m}$, an accelerator would need to be $100 \mathrm{~km}$ long to reach $10 \mathrm{TeV}$ and would be cost prohibitive to build.

Accelerators of high-energy particles have many applications beyond elementary particle research, for example, medical therapy [2], sterilization of material, and radioisotope production. Intense, continuously tunable radiation from $\mathrm{x}$ rays to millimeter waves is also available as a result of accelerator technology [3]. This has applications in biological and materials research [4] and industrial processing [5]. These applications would greatly benefit by having more compact, less expensive accelerators [6], which can deliver particle beam energies of hundreds of $\mathrm{MeV}$ to $\sim 1 \mathrm{GeV}$. This implies the need for increasing the acceleration gradient of these devices.

*Electronic address: wkimura@stioptronics.com
One possible key to the development of advanced accelerators is the usage of high-power lasers during the acceleration process to achieve high acceleration gradients. Laser accelerators have demonstrated gradients of $>100 \mathrm{GeV} / \mathrm{m}$ [7]; however, thus far this has been limited to distances from $<1 \mathrm{~mm}$ to several millimeters. Acceleration of particle beams to very high energies requires staged systems, whereby the particle beam interacts repeatedly with the laser beam and is guided and accelerated in an organized fashion through each stage of ever increasing particle beam energy. This, in turn, requires that the laser and particle beams are synchronized to a precision of the order of 1 optical wavelength.

To be more precise, demonstration of staged acceleration by a short wavelength driver, such as a laser, has three critical requirements.

(i) The particle beam must be organized into one or more very short bunches with bunch lengths a fraction of the laser wavelength. For a train of bunches, they must also be spaced apart at exactly the laser wavelength. In the case of the $\mathrm{CO}_{2}$ laser $(\lambda=10.6 \mu \mathrm{m})$ used in the experiment described here, this requires an $e$-beam bunch length of $\sim 1 \mu \mathrm{m}$ or less with the bunches spaced apart by $10.6 \mu \mathrm{m}$. These ultrashort electron microbunches are equivalently only several femtoseconds in time duration. Thus, the function of the first laser-driven stage, called the buncher, 
is to create these microbunches by modulating the energy of the $e$ beam.

(ii) The system must be designed to minimize lengthening out of the microbunches (bunch smearing) while traveling through the beam line. Bunch smearing can be caused by unequal path lengths taken by electrons within the microbunches and/or by space charge spreading apart the electrons. Requirements (i) and (ii) comprise the "injection" process, whereby the electrons are grouped together in a manner that permits them to be injected into subsequent laser acceleration stages located downstream of the buncher.

(iii) Staging requires control and stability to femtosecond accuracy of the microbunch synchronization with the laser wave inside the subsequent laser-driven stages (i.e., accelerators). This process requires phase synchronization whereby the microbunches are confined to a limited portion of the laser wave as the electrons travel through the accelerator. By achieving the proper position on the wave, these electrons can be accelerated together as a group while maintaining a small energy spread.

This paper expands upon an earlier Letter [8] that describes the first achievement of all three requirements in an integrated experiment called STELLA (staged electron laser acceleration) located at the Brookhaven National Laboratory Accelerator Test Facility (ATF). We also demonstrated for the first time the ability to maintain stable femtosecond phase control and acceleration over a period of many minutes. While short microbunches have been generated before [9], during STELLA these microbunches were measured for the first time in a direct manner by detecting their energy distribution from which their bunch length was inferred.

We believe these results indicate that acceleration to ultrahigh energies in a laser-driven, staged accelerator will be possible in the future. Furthermore, this same technology may make feasible the development of compact, laserdriven accelerators.

\section{DESCRIPTION OF EXPERIMENT AND MODELING}

We briefly describe the experiment; further details can be found elsewhere $[8,10,11]$. Two identical inverse freeelectron lasers (IFELs) [12] are utilized as the buncher and accelerator. The IFELs used identical undulators manufactured by STI Optronics, consisting of a planar array of uniformly spaced permanent magnets with a magnet period of $3.3 \mathrm{~cm}$ and a total undulator length of $33 \mathrm{~cm}$. The electrons take oscillatory trajectories through the undulator, thereby introducing a transverse velocity component in the same direction of the laser field whose linear polarization is in the same plane as the electron undulation. At the proper resonance condition [13], the electrons stay synchronized with the optical field and continuously acquire net energy exchange.

The two IFELs are positioned in series and separated by $2.3 \mathrm{~m}$ along the beam line, as shown schematically in Fig. 1. A triplet is located before each IFEL to permit focusing the $e$ beam into the undulators. At the end of the beam line is an electron energy spectrometer for measuring the $e$-beam energy spectrum.

The pulsed ATF $\mathrm{CO}_{2}$ laser beam [14] is split into two beams with nominally $\approx 24 \mathrm{MW}$ peak power sent to the buncher (IFEL1) and 100-300 MW peak power sent to the accelerator (IFEL2). An adjustable optical delay stage in the laser beam transport to the accelerator provides gross path length control, and a rotating delay plate (not shown) allows fine phase adjustment $(<1 \mu \mathrm{m})$. This phase adjustment to a fraction of the laser wavelength is necessary to ensure the microbunches are synchronized to the maximum of the accelerating field.

The laser beams are converted to annular beams (not shown), are reflected collinear to the $e$-beam propagation by mirrors located inside the vacuum pipe (see Fig. 1), and are focused inside the undulators. These mirrors have a central hole to transmit the $e$ beam. The annular laser

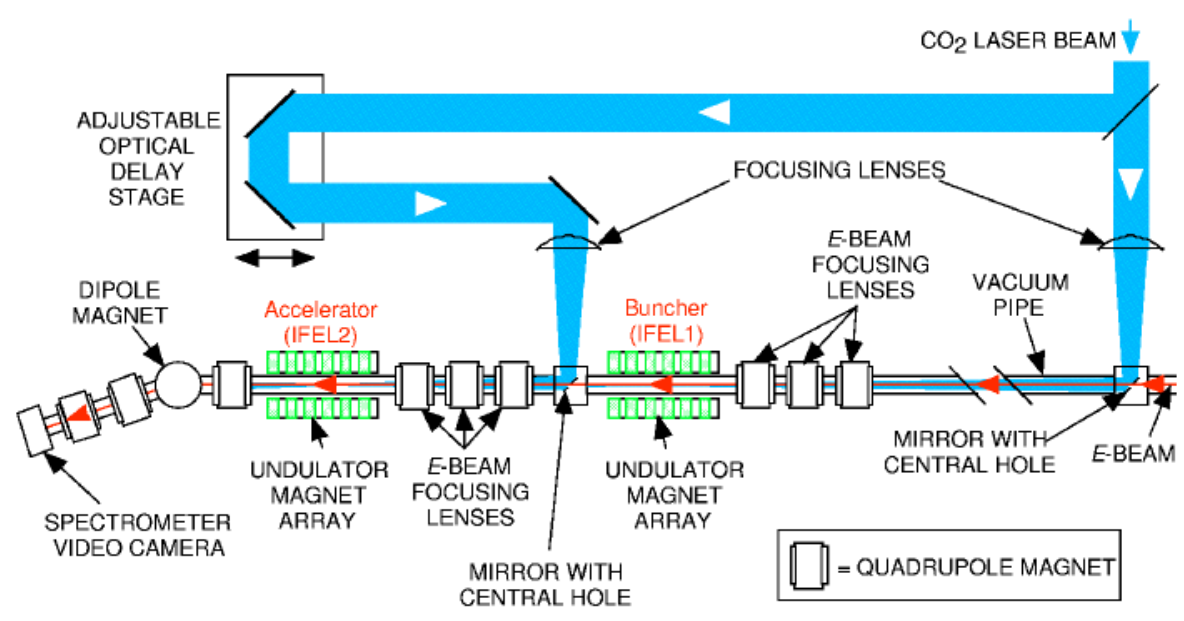

FIG. 1. (Color) Schematic layout for the STELLA experiment. 
TABLE I. STELLA experimental parameters and values used in the model.

\begin{tabular}{lc}
\hline \hline \multicolumn{1}{c}{ Parameter } & Value \\
\hline$e$-beam energy & $45.6 \mathrm{MeV}$ \\
$e$-beam intrinsic energy spread $(1 \sigma)$ & $0.04 \%$ \\
$e$-beam charge (total pulse) & $0.1 \mathrm{nC}$ \\
$e$-beam pulse length $(1 \sigma)$ & $\approx 3 \mathrm{ps}$ \\
$e$-beam normalized emittance & $1.5 \mathrm{~mm} \mathrm{mrad}$ \\
Laser pulse length (FWHM) & $\approx 180 \mathrm{ps}$ \\
Laser wavelength $\left(\mathrm{CO}_{2}\right.$ laser) & $10.6 \mu \mathrm{m}$ \\
Laser polarization & linear \\
Laser power to IFEL1 & $24 \mathrm{MW}$ \\
Laser beam size inside IFEL1 $(1 \sigma)$ & $0.67 \mathrm{~mm}$ \\
Laser power to IFEL2 & $100-300 \mathrm{MW}$ \\
Laser beam size inside IFEL2 $(1 \sigma)$ & $0.62 \mathrm{~mm}$ \\
$e$-beam angular error entering IFEL1 & $0.4 \mathrm{mrad}$ in $x$ and $y$ \\
$e$-beam centroid offset entering IFEL1 & $0.7 \mathrm{~mm}$ in $x$ only \\
\hline \hline
\end{tabular}

beams are focused into modified Airy patterns inside the undulators. The center of this pattern closely matches the Gaussian shape of the $e$ beam inside the undulator.

A 3D computer model of the STELLA experiment was developed (see the Appendix) that incorporates all effects including 1D longitudinal space-charge effects, $e$-beam emittance, and possible misalignment of the $e$ beam and laser beams along different parts of the staged beam line. In all the results shown in this paper the simulation used 5000 electrons, and the model and data energy spectra have been adjusted to have equal areas. The model tracks the evolution of these representative electrons through the experimental system and provides detailed information on the coordinates of each electron along the system. Thus, the model is especially useful because it can reveal characteristics about the electrons, which are not evident from the energy spectrum alone.

Actual experimental parameters are used in the model wherever possible. Intrinsic $e$-beam parameters, such as emittance, are chosen corresponding to the best agreement with the measured $e$-beam sizes along the beam line.

Table I lists the parameters for the STELLA experiment and the values used in the model comparisons given in this article.

\section{EXPERIMENTAL RESULTS AND DISCUSSION}

\section{A. Buncher output characteristics}

To begin this more detailed analysis of the experimental results we first examine the results for the buncher operating alone as given in Fig. 2. The graphs in this figure and others are shown with connecting dashed lines to facilitate understanding the relationship between the different plots. Figure 2(d) is the raw output from the spectrometer where energy dispersion is in the horizontal direction. A line profile through the center of this image is plotted (red curve) in Fig. 2(c) along with the energy spectrum histogram pre-

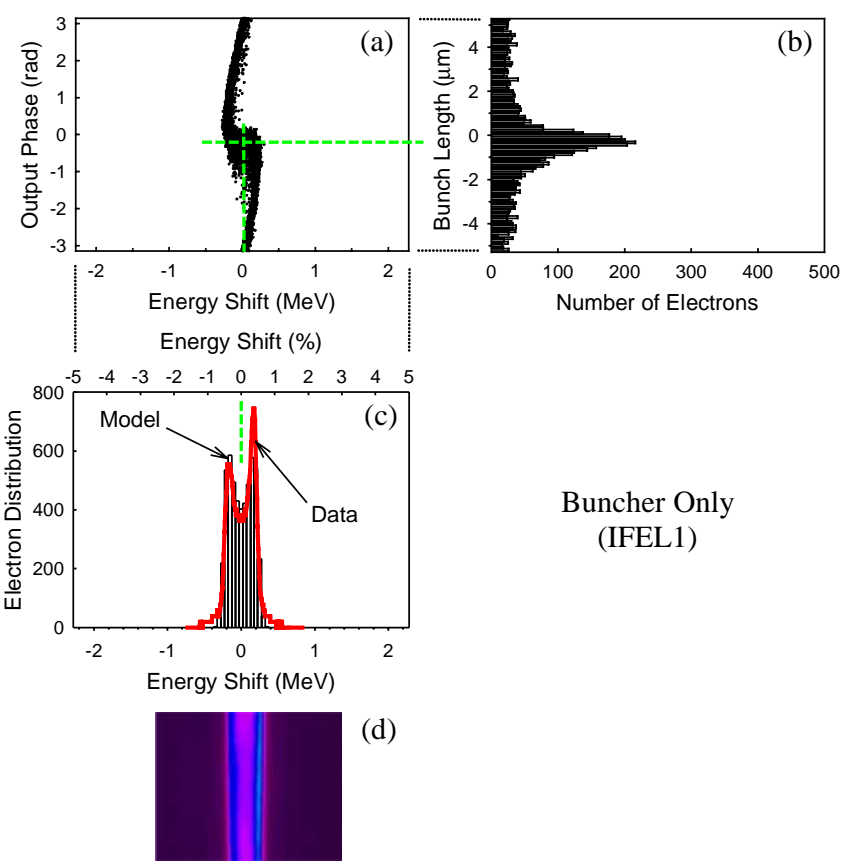

FIG. 2. (Color) Comparison of buncher only results with model. (a) Model-predicted electron output phase versus electron energy, (b) model-predicted electron bunch length, (c) electron energy spectrum, and (d) raw spectrometer output for data plotted in (c).

dicted by the model. Figure 2(c) shows the energy shift from the mean $e$-beam energy in absolute units and as a percentage of the mean $e$-beam energy $(45.6 \mathrm{MeV})$.

Both the data and the model display a symmetric, double-peaked energy spectrum. This is because the $3 \mathrm{ps}$ long $e$-beam pulse interacts over all phases of the laser field, which oscillates every 35 fs and has uniform intensity over the entire $e$-beam pulse length. This means the electrons experience varying amplitude and alternating polarity of the oscillating laser field, thereby resulting in some electrons being accelerated, some decelerated, and some with little energy change. If these electrons are allowed to drift, the accelerated electrons catch up with the decelerated ones, resulting in a bunching of a large number of electrons about a particular phase. This is in fact the microbunch formation described earlier as requirement (i). This process occurs routinely in microwavedriven accelerators; the complication in laser accelerators is that the bunch lengths are several orders of magnitude smaller.

This bunching effect can be seen more clearly in Fig. 2(a), which is the model prediction for the way in which the electrons in Fig. 2(c) are distributed in phase relative to the laser field after drifting $2.3 \mathrm{~m}$ downstream to the entrance of the accelerator. We see that there is a grouping of electrons centered around the $0 \mathrm{rad}$ phase. The distribution of these electrons within this grouping can be seen in Fig. 2(b), which plots the number of 
electrons as a function of their phase position. Note that, since $2 \pi$ of phase corresponds to $10.6 \mu \mathrm{m}$, Fig. 2(b) plots phase in the equivalent units of length. It indicates that the microbunch width entering the accelerator is $\approx 1.3 \mu \mathrm{m}$ (FWHM).

The drift distance required to achieve maximum bunch formation is controlled by the amount of laser power driving the buncher. Optical attenuators are used to adjust the laser power delivered to the buncher so that maximum bunching occurs approximately at the entrance to the accelerator. This corresponds to $\approx \pm 0.5 \%$ energy modulation [see Fig. 2(c)].

Since the 3 ps long $e$-beam pulse is $\approx 85$ optical wavelengths long, this means a train of $\approx 85$ microbunches is formed with each microbunch separated by $10.6 \mu \mathrm{m}$. In addition, since the diameter of the $e$ beam is of the order of several hundred microns, this implies the individual microbunches are pancake in shape with a diameter-tothickness aspect ratio of $>100: 1$. This is in sharp contrast to the long, cigar-shaped bunches generated in microwave accelerators, where the aspect ratio is typically reversed.

The pancake shape of microbunches also makes them more sensitive to space-charge spreading in the bunching direction rather than the transverse direction [15]. In microwave accelerators, space-charge spreading in the transverse dimension is usually more important.

\section{B. Staging results}

Figures 3-6 show typical results at the output of IFEL2 when operating both IFELs, i.e., during staging, at several different phase delays and their comparisons with the model. Figure 3 is at the phase delay corresponding to maximum acceleration. Figures $4-6$ are data taken at phase delays $90^{\circ}, 180^{\circ}$, and $310^{\circ}$, respectively, from Fig. 3. The model phase delay has been set to correspond to these same relative phases. For these simulations, the laser power driving the buncher and accelerator are 24 and 200 MW, respectively.

In Fig. 3(b), the model predicts that the bunch length is quite short $[\sim 0.8 \mu \mathrm{m}$ (FWHM) or $\sim 2.7 \mathrm{fs}]$. Shifting the phase by $90^{\circ}$, the microbunch moves to the center of the spectrum with some smearing of the bunch evident [Fig. 4(a)] and corresponding broadening of the bunch length [Fig. 4(b)]. In Fig. 4(c), two separate data profiles are plotted (red and blue curves), which were obtained many minutes apart to illustrate the reproducibility and stability of the data. This stability shows the submicron precision we were able to achieve during the experiment.

Shifting the phase by $180^{\circ}$, the microbunch is nearly fully decelerated [Fig. 5(c)]. Once again, a second data curve is shown taken many minutes apart from the first one. Note how the model predicts in Fig. 5(b) that the microbunch length has become more spread out, resulting in a decrease in the peak number of electrons.

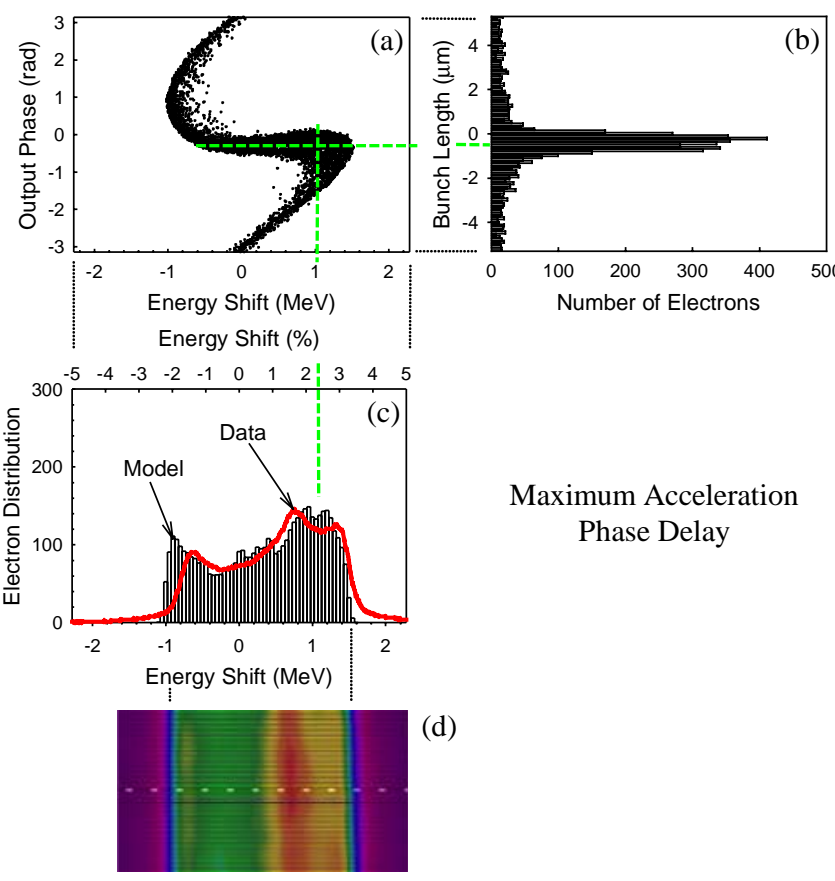

FIG. 3. (Color) Comparison of staging results with model for phase delay corresponding to maximum acceleration. (a) Model-predicted electron output phase versus electron energy, (b) model-predicted electron bunch length, (c) electron energy spectrum, and (d) raw spectrometer output for data plotted in (c).

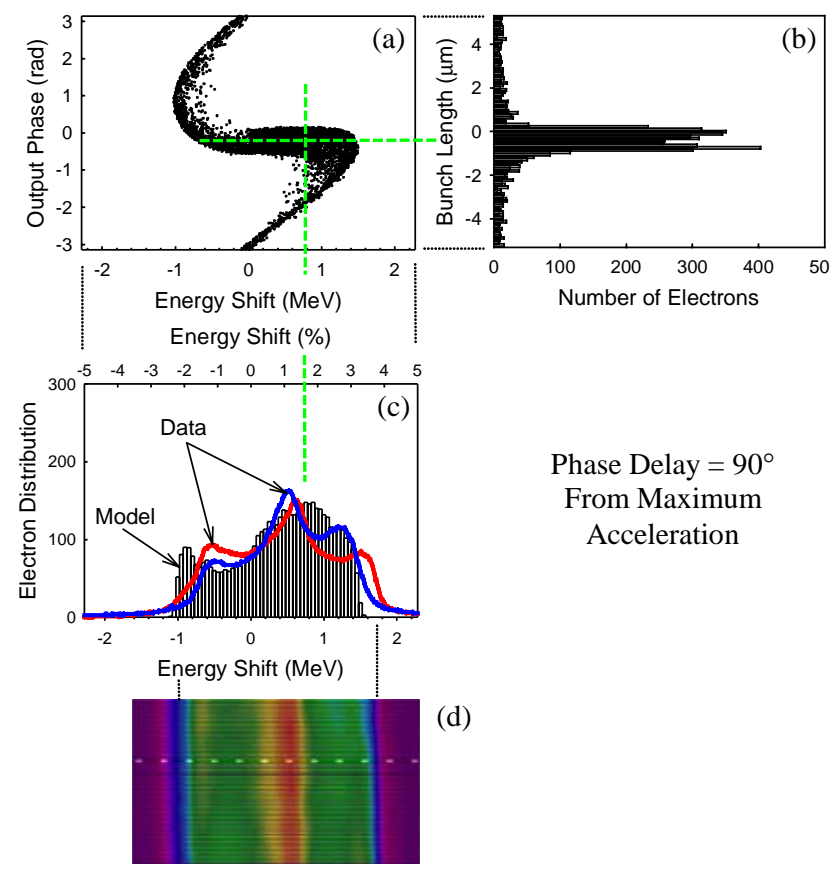

FIG. 4. (Color) Comparison of staging results with model for phase delay $90^{\circ}$ from Fig. 3. (a) Model-predicted electron output phase versus electron energy, (b) model-predicted electron bunch length, and (c) electron energy spectrum. Two separate data profiles are plotted (red and blue curves). (d) Raw spectrometer output for data plotted in (c). 


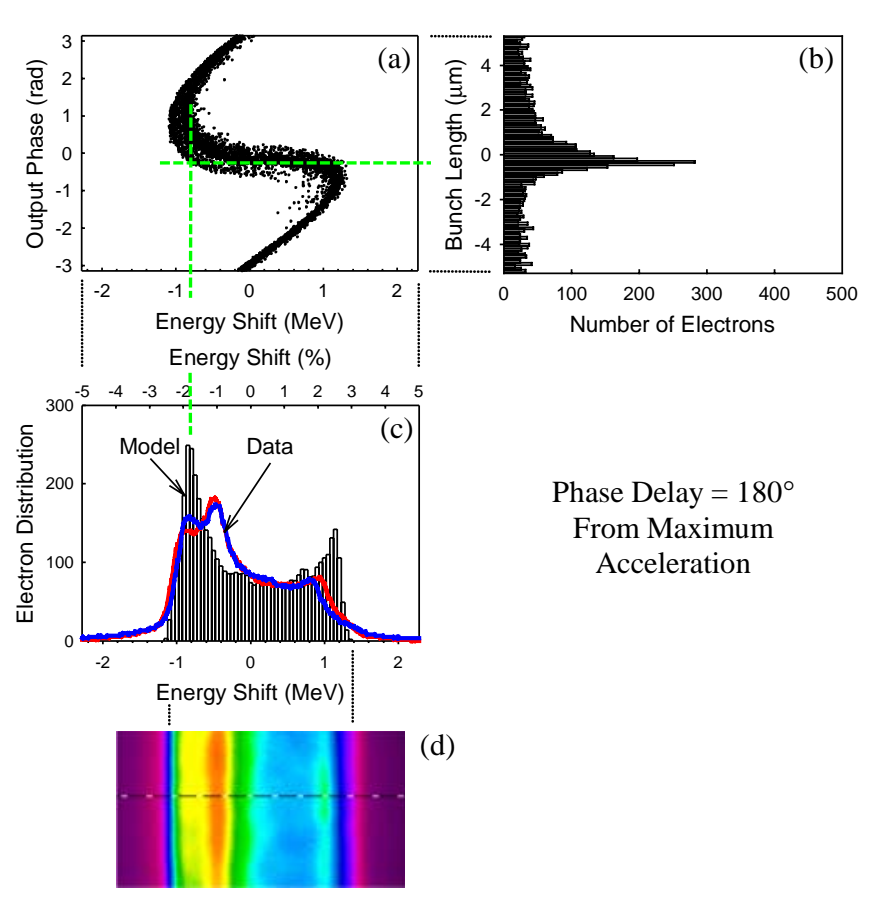

FIG. 5. (Color) Comparison of staging results with model for phase delay $180^{\circ}$ from Fig. 3. (a) Model-predicted electron output phase versus electron energy, (b) model-predicted electron bunch length, and (c) electron energy spectrum. Two separate data profiles are plotted (red and blue curves). (d) Raw spectrometer output for data plotted in (c).
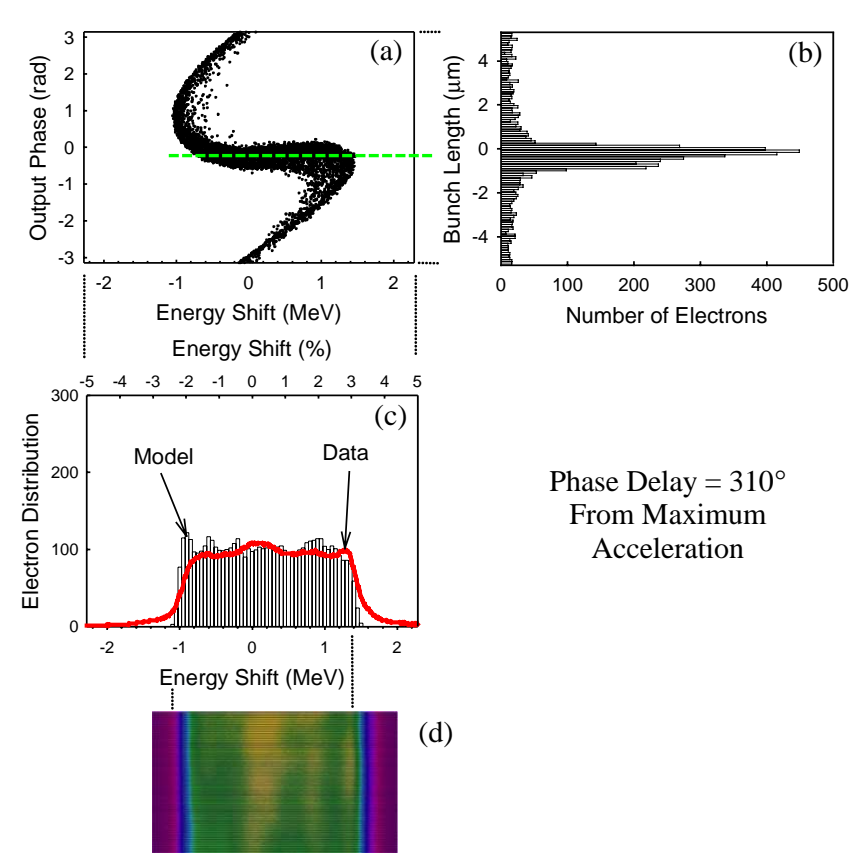

Phase Delay $=310^{\circ}$ From Maximum Acceleration

FIG. 6. (Color) Comparison of staging results with model for phase delay $310^{\circ}$ from Fig. 3. (a) Model-predicted electron output phase versus electron energy, (b) model-predicted electron bunch length, (c) electron energy spectrum, and (d) raw spectrometer output for data plotted in (c).
Finally, in Fig. 6 at $310^{\circ}$ the microbunch has once again reached an intermediate phase point, which is actually slightly over $180^{\circ}$ from Fig. 4, where it becomes stretched out in energy resulting in a remarkably flat energy spectrum. The agreement with the model is once again very good.

\section{Overmodulation in buncher results}

If too much laser power is sent to the buncher (so-called overmodulation), the electrons bunch to their minimum length before they reach the accelerator. They then begin to pass each other and the bunches smear away (debunch) when they reach the accelerator. The data and comparison with the model for an overmodulation case are given in Figs. 7 and 8 at two phase delays separated by $180^{\circ}$. The model parameters are similar to those used for Figs. 3-6, with the primary difference being that the laser power to the buncher and accelerator are 90 and $115 \mathrm{MW}$, respectively.

In Fig. 7(a) we see a distorted energy-phase distribution caused in part because the energy modulation is comparable in the buncher and accelerator. This also causes a corresponding spreading of the bunch length [Fig. 7(b)]. Because the microbunch is roughly centered in the energy spectrum, the spectrum simply displays a wider peak, which by itself would not necessarily indicate overmodulation. However, Fig. 8 shows that at a phase delay $180^{\circ}$ from Fig. 7 there is a distinct change in the energy
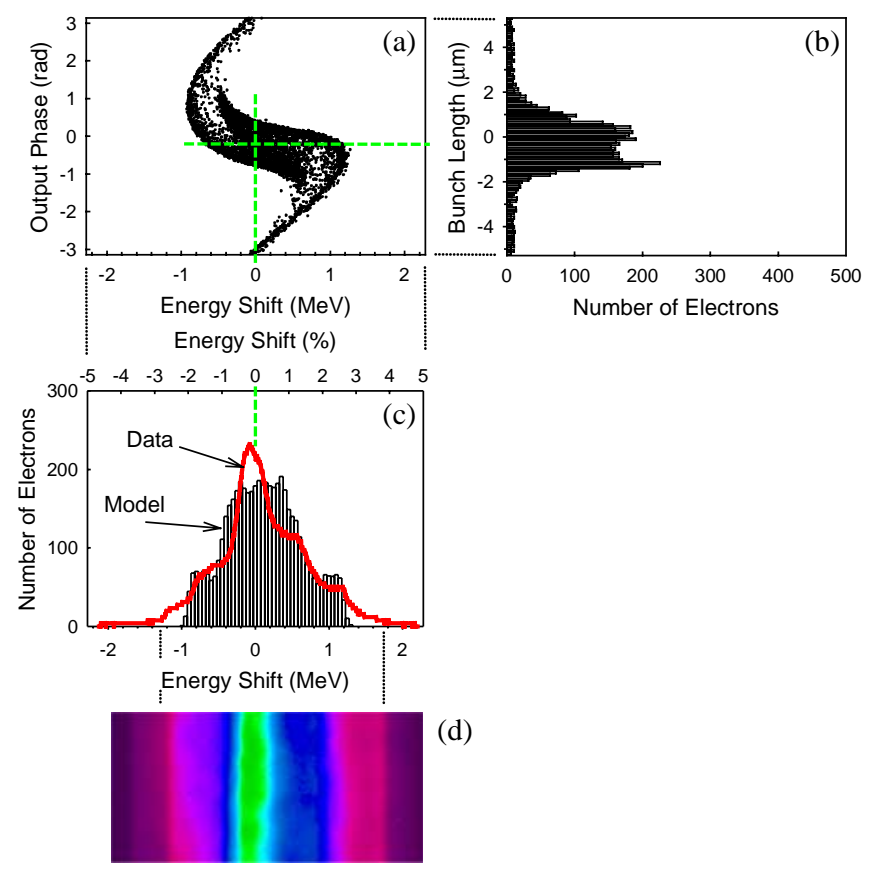

FIG. 7. (Color) Example of overmodulation in the buncher. (a) Model-predicted electron output phase versus electron energy, (b) model-predicted electron bunch length, (c) electron energy spectrum, and (d) raw spectrometer output for data plotted in $(c)$. 


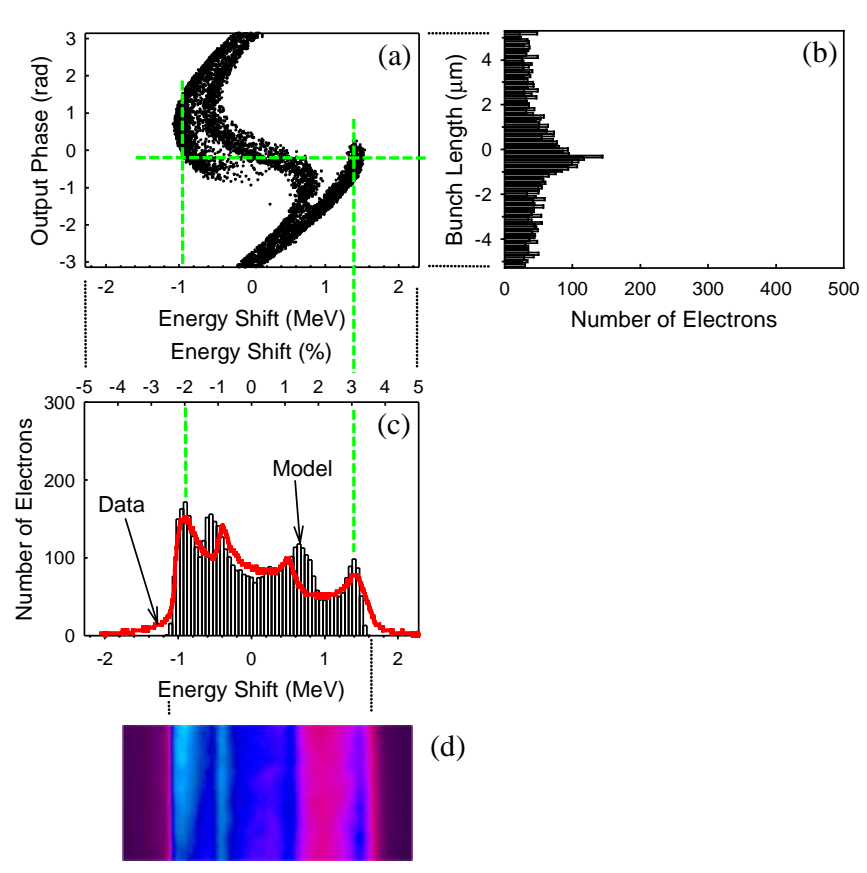

FIG. 8. (Color) Example of overmodulation in the buncher for phase delay $180^{\circ}$ from Fig. 7. (a) Model-predicted electron output phase versus electron energy, (b) model-predicted electron bunch length, (c) electron energy spectrum, and (d) raw spectrometer output for data plotted in (c).

spectrum [Fig. 8(c)], which is unlike that observed at optimum buncher modulation. At this phase, portions of the microbunch are simultaneously accelerated and decelerated resulting in a four-peaked spectrum. This characteristic spectral behavior is consistently reproduced experimentally when high laser intensity is intentionally applied in the buncher.

\section{General discussion of results}

The bunch length [see subfigures 2(b)-8(b)] can change throughout the system as the phase space evolves, i.e., the electrons shift in energy and phase position. For example, the microbunch length entering the accelerator [Fig. 2(b)] actually narrows somewhat after it is accelerated [Fig. 3(b)]. However, overmodulation can significantly redistribute the electrons in phase causing a widening of the bunch length [Fig. 7(b)] or destruction of the microbunch [Fig. 8(b)].

Trapping is the process whereby the electrons oscillate within a confined phase and energy range as they travel through an accelerator. The undulator in our accelerator is too short to permit multiple synchrotron oscillations to occur. However, the phase synchronization we have demonstrated should permit such trapping to occur in a practical laser accelerator.

The multiple data curves shown in Figs. 4 and 5 illustrate the ability to maintain stable phase control on the few femtosecond level over periods of up to many minutes.
This is noteworthy because in this particular experiment the laser beam injection points for the buncher and accelerator are physically separated by $6 \mathrm{~m}$, optical elements are secured on separate supports, the transport lines are not evacuated or tightly enclosed, and no active phase stabilization is used. The relatively long wavelength of the laser $(10.6 \mu \mathrm{m})$ helped enable this level of control by reducing the stability requirements of the optical transport system and precision needed for phase control.

Several experimental steps were taken to improve phase control. As mentioned previously, a rotating delay plate was used for fine phase adjustment because it permitted finer control than obtainable using the available stepper motors on the optical delay stage (see Fig. 1) and it was less prone to backlash errors. The large size of the experimental system with its many disparate components made it impractical to use thermally stabilized materials. Thus, attempts were made to minimize thermal drift by turning off the air conditioning in the room housing the beam line during the experiment. Other than these steps, the rest of the system used conventional linear accelerator and optical components for alignment, control, and diagnosis of the beams.

For an $e$-beam charge of $0.1 \mathrm{nC}$ during our experiment, the model indicates space charge effects do not appreciably smear out the microbunches. Angular misalignment of the $e$ beam through the triplet between the buncher and accelerator appears to have a much greater impact. Angular errors, where the $e$ beam is not perfectly centered and collinear on the beam line axis as it passes through the triplet, can exacerbate path length differences due to the intrinsic $e$-beam divergence. Divergence causes electrons at higher entrance angles to take longer trajectories than electrons traveling on axis. Path length differences of 1-2 $\mu \mathrm{m}$ can significantly interfere with the bunching process for a $10.6 \mu \mathrm{m}$ laser wavelength. Note that a shorter wavelength laser has a corresponding shorter path length difference tolerance.

As mentioned, whenever possible, actual experimental values were used in the model. Parameters that were adjusted are as follows:

(i) The laser power delivered to the undulators. Although the laser power is measured during the experiment, its peak power during each shot can vary due to changes in both the pulse energy and position of the $e$-beam pulse within the laser temporal profile. During the experiment we attempted to position the $e$-beam pulse near the top of the leading edge of the laser pulse, but not at the peak because the peak has the greatest tendency to vary from shot to shot.

(ii) The Twiss parameters of the $e$ beam entering the first undulator (see the Appendix). These parameters were adjusted to obtain the best agreement with the $e$-beam sizes measured at diagnostic ports along the entire STELLA beam line. Highest priority was given to obtaining a good agreement at the entrance to the second undulator 
(accelerator) since this is the position most sensitive to the staging process and where space charge effects would be the strongest.

(iii) $e$-beam angular error and centroid offset entering the first undulator (see Table I). During the experiment, there was a limit to how accurately the $e$ beam and laser beams could be overlapped and made collinear in their propagation. While the model has an option to include angular error and centroid offset entering both undulators, only errors and offsets for the $e$ beam entering IFEL1 were adjusted. This is not to imply that such errors and offsets did not exist for the $e$ beam entering IFEL2; however, we found that very good agreement could be obtained by simply adjusting these parameters for IFEL1. One could also argue that such errors and offsets for IFEL2 would have much less affect on the microbunches entering IFEL2 because the microbunch formation and position in phase is already determined by the time they reach IFEL2. Surprisingly small angular errors $(\sim 400 \mu \mathrm{rad})$ and offsets $(\sim 200-700 \mu \mathrm{m})$ had a significant affect on details of the energy spectra predicted by the model. These details were primarily the position and magnitude of the various peaks seen in the spectra, which are caused by changes in the position and grouping of the microbunch in energy-phase space. Again, submicron movement of the microbunch can cause the peak in the energy spectra, which denotes the microbunch presence, to shift within the spectra. While these angular errors and offsets are only estimates and could not be directly measured, the fact that good agreement was obtained at different phase delays and conditions while keeping the values for these errors and offsets in the model nearly unchanged is strong evidence supporting the validity of this approach.

Maximum energy gains in the accelerator of $\approx 5 \%$ were observed corresponding to an acceleration gradient of nearly $7 \mathrm{MeV} / \mathrm{m}$, which is modest compared to other laser acceleration experiments where much higher gradients have been demonstrated [7]. We emphasize that high gradient acceleration was not the primary goal of STELLA, but rather the staging of two laser-driven systems. In addition, we note the second undulator uses an untapered geometry, which limits its maximum attainable acceleration.

\section{E. Monoenergetic laser acceleration (STELLA-II)}

A program to demonstrate monoenergetic laser acceleration is currently underway, which we call STELLA-II. STELLA-II builds upon the success of STELLA and addresses two important issues. First, a single laser beam will drive both the buncher and accelerator during STELLA-II. This greatly reduces phase jitter related to using separate laser beams to drive the IFELs. This new system is depicted in Fig. 9.

Second, reducing the energy spread of the microbunches during the acceleration process can be accomplished only by trapping the microbunches in the accelerators. The untapered undulator of STELLA prevented this trapping. A tapered undulator will be used for the accelerator during STELLA-II. Together with the substantially higher laser power that will be available from the ATF $\mathrm{CO}_{2}$ laser, this will enable trapping of the microbunches and separating in energy the microbunches from the untrapped background electrons.

As an example, with $100 \mathrm{GW}$ of laser power and $25 \%$ taper in the accelerator undulator, the model predicts an energy gain of $\approx 13 \mathrm{MeV}$ for a $45.6 \mathrm{MeV} e$ beam. It predicts the accelerated microbunch will have an energy spread of $\approx 1.2 \%$ FWHM with a capture efficiency of $\sim 80 \%$. These predictions are shown in Fig. 10.

STELLA-II will be using the upgraded ATF laser [16] capable of delivering several hundred gigawatts of peak power in the near term and eventually $\sim 1 \mathrm{TW}$. Because this laser power is much larger than needed to drive the buncher, the undulator for the buncher will be replaced with a three-period electromagnet that is intentionally detuned in order to still provide a modulation of $\pm 0.5 \%$ even with hundreds of GW of laser power passing through it.

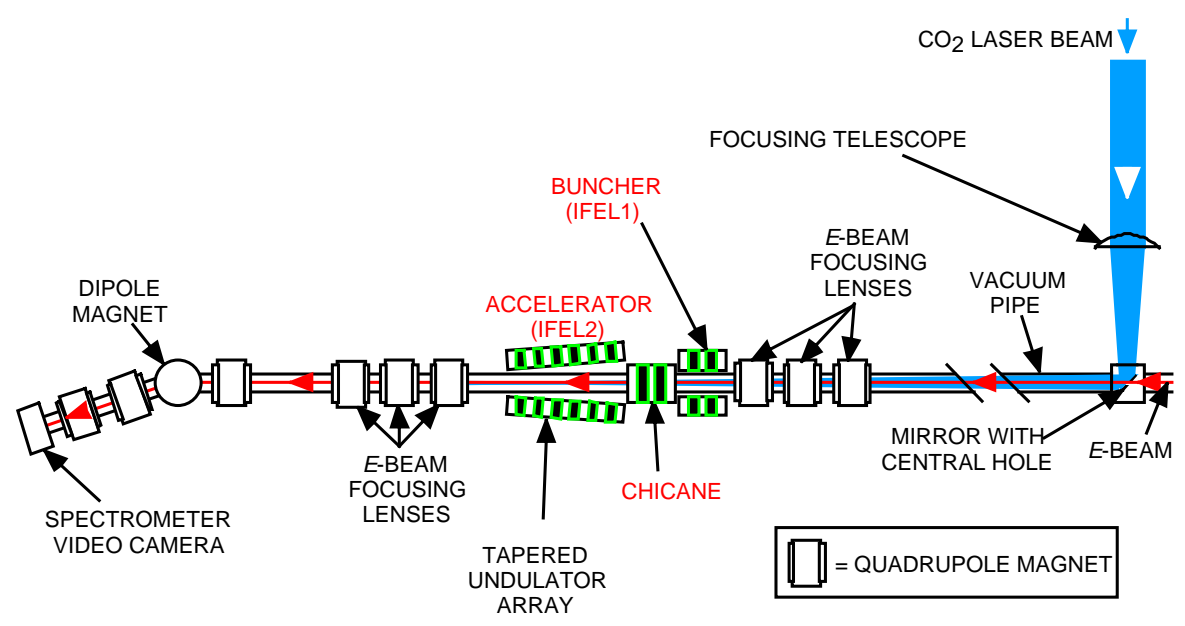

FIG. 9. (Color) Schematic layout for the STELLA-II experiment. 


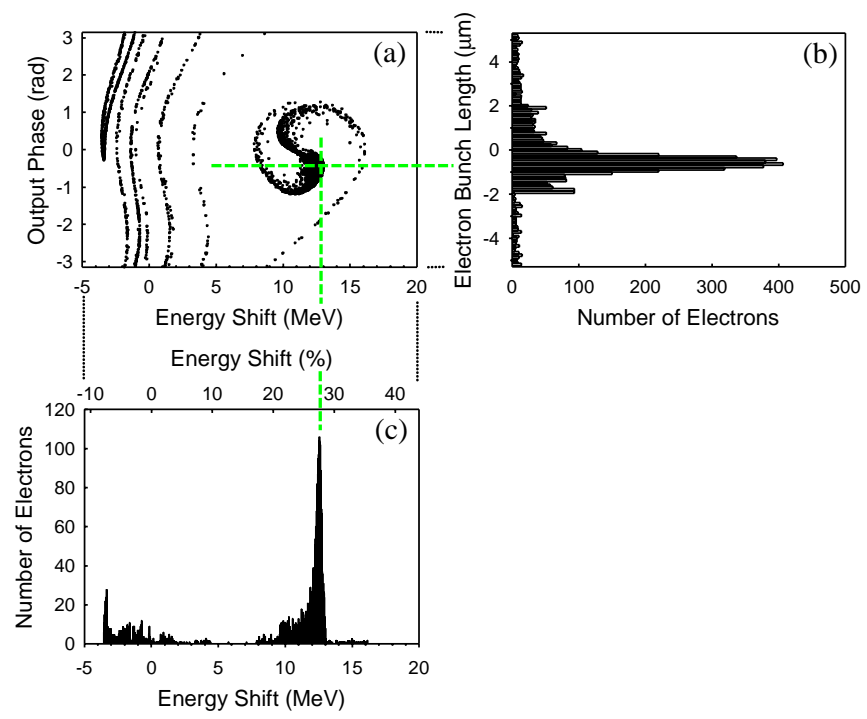

FIG. 10. (Color) Model predictions for STELLA-II for $100 \mathrm{GW}$ laser power and $25 \%$ taper in the accelerator undulator. (a) Model-predicted electron output phase versus electron energy, (b) model-predicted electron bunch length, and (c) model-predicated electron energy spectrum.

Using a single laser also requires a minimum drift space between the IFELs. Thus, a short-length magnetic chicane will be utilized between the new buncher and the tapered accelerator. The laser beam will also be passing through the chicane. Thus, to minimize the possibility of additional laser-induced modulation occurring, the chicane will be oriented with its magnetic field orthogonal to the buncher.

Hence, STELLA-II will demonstrate monoenergetic acceleration of the microbunches. Although the optimum bunch length and energy spread for a practical staged laser accelerator system still needs to be determined, this next experiment is another important step toward the goal of eventually building practical laser accelerators and enabling femtosecond electron microbunches created in this manner to be used for other applications.

\section{CONCLUSIONS}

A number of noteworthy accomplishments occurred during the STELLA program: (i) the first demonstration of a laser-driven buncher staged together with a laser-driven accelerator; (ii) the first direct measurement of $\sim 3$ fs microbunches produced by a laser external to an undulator; (iii) the first demonstration of acceleration of lasergenerated microbunches with stable phase control maintained over periods of many minutes; and (iv) the first demonstration of laser-accelerated microbunches where a large portion of the electrons receive maximum energy gain. This last accomplishment is particularly noteworthy since the accelerated electrons in laser acceleration experiments to date typically exhibit wide energy distributions with only a relatively small number of electrons experiencing a narrow energy gain.

The close agreement between the data and the model implies that the experimental results are a product of a systematic approach and thorough understanding of the process; the microbunch behavior is understood at every stage and phase, and the model can be confidently used for further predictions and optimization.

The experiment also showed the benefits of using a long wavelength laser, which eases stability and rephasing requirements, and lessens sensitivity to bunch smearing effects.

\section{ACKNOWLEDGMENTS}

The authors wish to acknowledge Dr. J. R. Fontana for his technical advice during the initial planning of this experiment, and Dr. Xijie Wang and the staff at the ATF for their support. This work was supported by the U.S. Department of Energy, Grants No. DE-FG03-98ER41061, No. DE-AC02-98CH10886, and No. DE-FG03-92ER40695.

\section{APPENDIX: DESCRIPTION OF STELLA MODEL}

A simulation model is used to aid in performance predictions and sensitivity studies for the STELLA experiment. The code simulates the free-electron laser (FEL) interaction in the buncher and accelerator stages and includes a particle-tracking model for the electron paths through the intervening drift region. This appendix describes the major features of the model.

The FEL model includes a full 3D implementation permitting the sensitivity to emittance and misalignment effects to be determined. Possible bunch smearing in the drift region is studied by consideration of longitudinal space charge spreading as well as the path length differences due to finite beam size and misalignments. The STELLA model is kept as simple and flexible as possible to allow for rapid changes when the experimental configuration is modified or important new physical effects are to be considered.

As shown schematically in Fig. 11, the integrated STELLA model presently consists of two modules: an FEL model and a drift region model. The $e$ beam is modeled using typically 5000 simulation particles. Electron parameters in longitudinal and transverse phase space are passed between modules when needed. Modules are run repeatedly as needed to simulate staged systems. The FEL module is initiated with the electron distribution provided by the linear accelerator. It then calculates the energy modulation induced by the FEL interaction in the buncher. The drift region model then computes the microbunching resulting from the induced energy spread, including any bunch smearing effects. The FEL module is then reused to simulate the acceleration of the bunched beam. 


\section{Experimental System}
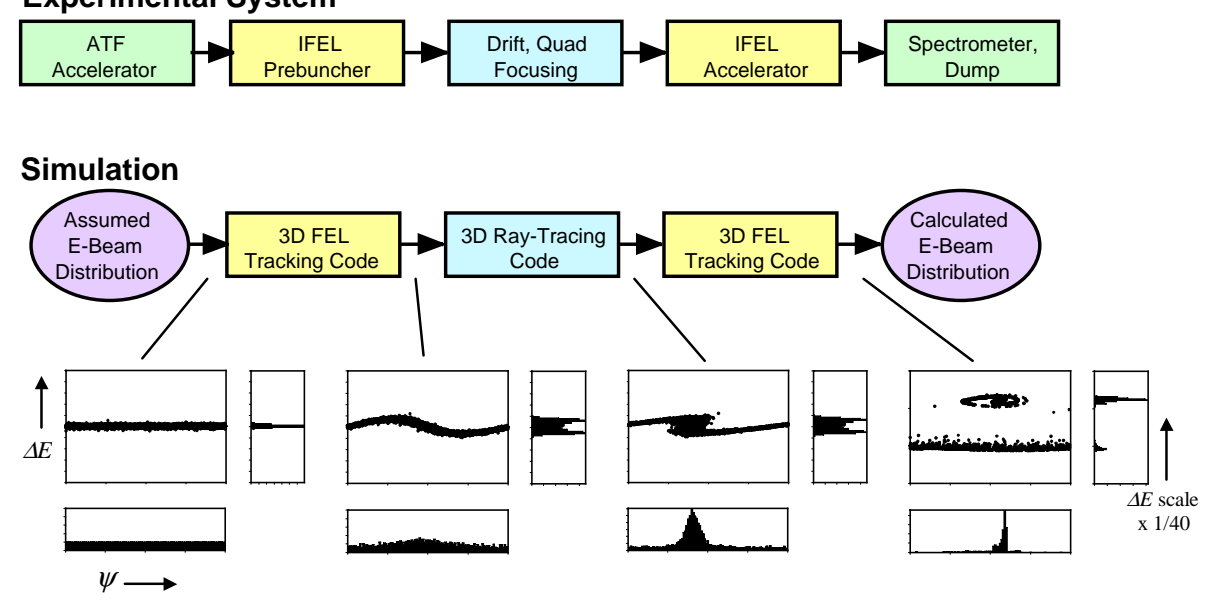

FIG. 11. (Color) Schematic flow chart of the integrated STELLA model.

\section{A. FEL simulation model}

The 3D FEL model is based on classical wiggleaveraged FEL theory, i.e., the pendulum equation [17]. In practice, the $e$ beam is modeled by setting the effective emittance and Twiss parameters to approximately match the experimentally observed beam sizes along the beam line. Since the laser pulse $(\sim 180 \mathrm{ps})$ is much longer than the $e$-beam pulse ( $\sim 3 \mathrm{ps}$ ), the entire $e$ beam is assumed to be illuminated with uniform laser power. Thus, it is sufficient to model the behavior of a single $e$-beam microbunch within the train of microbunches inside the overall $e$-beam envelope. The laser beam is approximated as a cylindrically symmetric Gaussian-spherical TEM 00 mode. This profile is similar to the center of the modified Airy pattern when the annular laser beams are focused in the undulators. For simplicity, the effect of the $e$ beam on the laser beam (gain/loss and phase shift) is neglected. This approximation is reasonable for present moderate laser power experiments $($ loss $<10 \%)$ and will become even more valid when higher laser power is available in the near future.

A description of the $e$ beam and laser beams delivered to the entrance to the first undulator (buncher) initiates the simulation. The initial electron distribution function is characterized in terms of energy spread and emittance. The energy spread distribution is assumed to be Gaussian, with the form $\exp \left[-\left(\gamma-\left\langle\gamma_{0}\right\rangle\right)^{2} / 2 \sigma^{2}\right]$, where $\gamma$ is the electron energy (in units of the rest mass $m c^{2}$ ) and $\sigma$ is the standard deviation. The transverse emittance phase space is also filled with Gaussian distributions characterized by standard deviations $\sigma_{x}, \sigma_{y}, \sigma_{x}^{\prime}, \sigma_{y}^{\prime}$ in each of the four emittance coordinates. In general, the normalized rms emittance in the transverse plane denoted by the $x$ coordinate is given by [18]

$$
\varepsilon_{n x(\mathrm{rms})}=4 \gamma \sqrt{\left\langle(x-\langle x\rangle)^{2}\right\rangle\left\langle\left(\theta_{x e}-\left\langle\theta_{x e}\right\rangle\right)^{2}\right\rangle-\left\langle(x-\langle x\rangle)\left(\theta_{x e}-\left\langle\theta_{x e}\right\rangle\right)\right\rangle^{2}} .
$$

Here the angle brackets denote averages over the entire particle distribution. The quantity $\theta_{x e}$ is the transverse angle of an electron trajectory relative to the undulator axis. When the $e$ beam is at a waist, this simplifies to $\varepsilon_{n x(\mathrm{rms})}=4 \gamma \sigma_{x} \sigma_{x}^{\prime}$, where $\sigma_{x}$ and $\sigma_{x}^{\prime}$ are the $1 \sigma$ values for the $e$-beam size and angular divergence in that plane, respectively. (We note that the convention ordinarily used at the ATF is to quote measured normalized emittance in terms of $\varepsilon_{n x(\mathrm{ATF})}=\gamma \sigma_{x} \sigma_{x}^{\prime}$, which is onefourth of the rms emittance. The unnormalized form of this emittance, i.e., $\varepsilon_{x(\mathrm{ATF})}=\sigma_{x} \sigma_{x}^{\prime}$, is the form used for the usual Twiss parameter description of the beam envelope [19], i.e., $\sigma_{x}^{2}=\beta_{x} \varepsilon_{x(\mathrm{ATF})}$, where $\beta_{x}$ is the $x$-plane beta function Twiss parameter.)

The transverse emittance phase space is initiated at the entrance to the first stage according to input values for the alpha and beta functions (Twiss parameters), $\alpha_{x}, \alpha_{y}$, $\beta_{x}$, and $\beta_{y}$. These values are typically selected to approximately match the $e$-beam envelope within the undulator focusing channel and for best overlap with the laser beam. Minor adjustments about the ideal matching conditions may be applied to account for deviations observed based on experimentally measured $e$-beam sizes at the diagnostic stations along the beam line. At the entrance to the second or subsequent stages the transverse emittance phase space is simply handed off between the various modules of the simulation code without further manual adjustments.

Each simulation particle is tracked using the following equations for the spatial evolution of the electron energy $\gamma$ and phase $\psi$ relative to the optical wave [20]: 


$$
\frac{d \gamma}{d z}=\frac{-G e_{s}(x, y, z) a_{w}(y, z) \sin [\psi+\phi(x, y, z)]}{\gamma},
$$

$$
\frac{d \psi}{d z}=k_{w}(z)-\frac{k_{s}}{2 \gamma^{2}}\left[1+a_{w}^{2}(y, z)+\gamma^{2}\left(\theta_{x e}^{2}+\theta_{y e}^{2}\right)\right] .
$$

Here $z$ is the longitudinal coordinate (along the planarundulator axis) and $(x, y)$ are the transverse coordinates (wiggle direction and $B$-field gradient direction, respectively). The FEL interaction term on the right-hand side of Eq. (A2) includes the usual planar-undulator coupling factor, $G=J_{o}(A)-J_{1}(A), A=a_{w}^{2} / 2\left(1+a_{w}^{2}\right)$, given by the difference between two Bessel function terms. Laser beam electric field amplitude $E_{o}$ is represented by the normalized rms quantity $e_{s}(x, y, z)=e E_{o}(x, y, z) / \sqrt{2} m c^{2}$, where $e$ is the electron charge. The undulator field am-

plitude $B_{o}$ is represented by the dimensionless rms vector potential $a_{w}(y, z)=e B_{o}(z) \cosh \left(k_{w}(z) y\right) / \sqrt{2} k_{w}(z) m c$ (MKS units), where $k_{w}(z)=2 \pi / \lambda_{w}(z)$ is the undulator wave number. The undulator $B$ field and period $\lambda_{w}$ are generalized to include axial dependence to allow for simulation of tapered-undulator operation. Note that the $\cosh k_{w} y$ transverse dependence of the planar undulator field is included. The phase of the optical beam $\phi(x, y, z)$ represents the deviation of the laser beam phase relative to an ideal forward-directed plane wave. The optical field wave number is $k_{s}=2 \pi / \lambda_{s}$, where $\lambda_{s}$ is the laser wavelength. Higher harmonics of the optical field are neglected.

The spatial distribution of a fixed $\mathrm{TEM}_{00}$ laser beam is given by [21]

$$
e_{x}(x, y, z)=e_{s o}\left(\frac{w_{o}}{w(z)}\right) e^{-\left(\left\{\left[x-x_{o}(z)\right]^{2}+\left[y-y_{o}(z)\right]^{2}\right\} / w^{2}(z)\right)}
$$

$$
\begin{aligned}
\phi(x, y, z)= & \left(\frac{\left[x-x_{o}(z)^{2}\right]+\left[y-y_{o}(z)\right]^{2}}{w^{2}(z)}\right)\left(\frac{z-z_{w}}{z_{R}}\right)-\tan ^{-1}\left(\frac{z-z_{w}}{z_{R}}\right)+k_{s}\left(\frac{\theta_{x o}^{2}+\theta_{y o}^{2}}{2}\right) z \\
& +k_{s}\left(x-x_{o}(z)\right) \theta_{x o}+k_{s}\left(y-y_{o}(z)\right) \theta_{y o},
\end{aligned}
$$

where $e_{s o}$ is the peak $E$ field at the beam waist, $w_{o}$ is the $1 / e$ amplitude radius at the waist, $z_{R}$ is the Rayleigh range, $z_{w}$ is the axial position of the beam waist, $w(z)=$ $w_{o} \sqrt{1+\left(z-z_{w}\right)^{2} / z_{R}^{2}}$ is the spatial dependence of the beam radius, $x_{o}(z)=x_{o}(0)+\theta_{x o} z$ and $y_{o}(z)=y_{o}(0)+$ $\theta_{y o} z$ are the transverse positions of the laser beam centroid, and $\theta_{x o}$ and $\theta_{y o}$ are the angles of the laser beam propagation relative to the undulator axis. Note that the optical phase in Eq. (A5) consists of five terms representing (a) the transverse wave front curvature, (b) the on-axis phase shift resulting from the curvature (Guoy phase shift), (c) the term describing any Doppler shift due to propagation at an angle, (d) any wave front tilt in the $x$ direction, and (e) any wave front tilt in the $y$ direction.

These equations, together with a solution to the equations for the trajectories of each simulation electron

$$
\begin{gathered}
\frac{d x}{d z}=\theta_{x e}, \\
\frac{d y}{d z}=\theta_{y e}, \\
\frac{d \theta_{x e}}{d z}=0, \\
\frac{d \theta_{y e}}{d z}=-k_{\beta}^{2} y,
\end{gathered}
$$

provide a complete description of the $e$-beam dynamics in the FEL region. Here we include $y$-direction $e$-beam focusing [22] due to interaction with the planar-undulator magnetic field, where $k_{\beta}=a_{w} k_{w} / \gamma$ is the betatron wave number. By including all key transverse-dependent effects on the FEL interaction, the detuning resulting from both effective energy spread due to emittance and loss of overlap is included directly in the simulation. Thus the 3D model can predict the sensitivity to emittance and misalignment.

\section{B. Drift region model}

In the drift region of the IFEL buncher, the time of flight of each electron depends on its energy and trajectory. The optical phase of an electron at the end of a drift region may differ from that of a reference resonant on-axis electron as a result of contributions from energy variance and off-axis path length difference. Modeling for the parameters of interest shows that significant bunch smearing effects are possible if care is not taken to avoid large emittance or misalignments.

Bunch smearing is modeled by tracing the individual trajectories of a large number of simulation electrons distributed throughout emittance phase space. Trajectories are calculated in a manner analogous to Eqs. (A6)-(A9), but including the interaction with the magnetic field of quadrupoles located between the buncher undulator and the second (accelerator) stage. The axial velocity $\beta_{z}=\nu_{z} / c$ of a relativistic electron is

$$
\beta_{z}=1-\frac{1}{2 \gamma^{2}}\left[1+\gamma^{2}\left(\theta_{x e}^{2}+\theta_{y e}^{2}\right)\right]
$$

in the small angle approximation. Optical phase change due to energy differences relative to that of a resonant on-axis electron of energy $\gamma_{r}$ is 


$$
\Delta \psi=\frac{\pi L_{D}}{\lambda_{s}}\left(\frac{1}{\gamma_{r}^{2}}-\frac{1}{\gamma^{2}}\right),
$$

where $L_{D}$ is the total drift length. For equal electron energies, the increased path length of an off-axis electron over a drift length $L_{D}$ is given by

$$
\Delta d=\frac{1}{2} \int_{0}^{L_{D}}\left(\theta_{x e}^{2}+\theta_{y e}^{2}\right) d z
$$

The path length contribution to optical phase difference $\Delta \psi=-2 \pi \Delta d / \lambda_{s}$ when combined with the energy variance contribution yields

$$
\Delta \psi=\frac{\pi L_{D}}{\lambda_{s}}\left(\frac{1}{\gamma_{r}^{2}}-\frac{1}{\gamma^{2}}\right)-\frac{\pi}{\lambda_{s}} \int_{0}^{L_{D}}\left(\theta_{x e}^{2}+\theta_{y e}^{2}\right) d z,
$$

relative to a resonant on-axis electron. The integral term in Eq. (A13) is computed by summation of the individual contributions from the various segments of the drift region, including quadrupoles and intervening drift segments.

\section{Space charge effects}

It has been shown [15] that longitudinal space charge can significantly affect the degree of microbunching for parameters similar to those of the present STELLA experiments. In order to include this effect in the numerical simulations, the following simple 1D longitudinal space charge model is included in the drift region simulation.

The gross axial space charge $E$ field is determined based on a simplified analytic model and this field is then included in the Lorentz force in the equations of motion. The approach is to find the 1D solution of the Gauss equation for a Gaussian fit to the calculated axial electron phase distribution. Two-dimensional correction effects are applied a posteriori.

The 1D Gauss equation in MKS units is

$$
\frac{d E_{z}}{d z}=-\frac{e}{\varepsilon_{o}} n_{e}(z)
$$

where $\varepsilon_{o}$ is the permittivity of free space. The nominal on-axis electron density $n_{e o}$ for a beam of current $I$, Gaussian transverse profile, and transverse beam sizes $(1 \sigma)$ in each plane, $\sigma_{x}$ and $\sigma_{y}$, is given by

$$
n_{e o}=\frac{I}{2 \pi e c \sigma_{x} \sigma_{y}} .
$$

Supposing that the axial electron density in a single microbunch also takes the form of a Gaussian distribution superposed on a uniform background distribution,

$$
n_{e}(\psi)=n_{e o}\left(f_{o}+f_{b} \exp \left[-\left(\psi-\psi_{b}\right)^{2} / 2 \sigma_{b}^{2}\right]\right),
$$

where $n_{e o}\left(f_{o}+f_{b}\right)$ is the peak (center) density in the microbunch, $f_{o}$ is the fraction due to the background, and $f_{b}$ is the fraction attributable to the microbunch. Here we express the longitudinal coordinate using the phase $\psi=$ $z k_{s}$ (radians), where $2 \pi / k_{s}$ is the spatial wavelength of the bunching, $\psi_{b}$ is the phase of the bunch center, and $\sigma_{b}$ is the Gaussian standard deviation (radians) of the bunch. In the numerical model, the parameters expressing the degree of microbunching are found by a least squares fit of the calculated instantaneous density distribution $n_{e}$ versus $\psi$ to the expression given in Eq. (A16), based on all simulation particles within the $2 \pi$ window. Using this fit has the beneficial effect of smoothing out statistical deviations in the charge density due to the relatively few number of simulation particles.

The solution to Eq. (A15), assuming zero electric field at the bunch center, is

$$
\left(E_{z}\right)_{1 \mathrm{D}}=-\sqrt{\frac{\pi}{2}} \frac{e n_{e o} f_{b}}{\varepsilon_{o}} \frac{\sigma_{b}}{k_{s}} \operatorname{erf}\left[\left(\psi-\psi_{b}\right) / \sqrt{2} \sigma_{b}\right],
$$

where erf is the error function. The assumption that the field is zero at the microbunch center ignores the space charge effects of the other microbunches as well as the unbunched electrons strewn in between. This is justified because only the electrons in the microbunch itself produce significant electric field gradients that result in space charge debunching.

The two-dimensional correction $F_{2 \mathrm{D}}$ derived in [15] can be used,

$$
E_{z}=\left(E_{z}\right)_{1 \mathrm{D}} F_{2 \mathrm{D}}=\left(E_{z}\right)_{1 \mathrm{D}} /(1+0.58 A)^{2},
$$

where the relativistically corrected aspect ratio of the microbunch shaped "pancake" (see discussion in main text) is

$$
A=\langle\gamma\rangle \sigma_{b} / k_{s} \sqrt{\sigma_{x} \sigma_{y}},
$$

and $\langle\gamma\rangle$ is the average relativistic factor of the beam.

For the purposes of the numerical model we make one additional correction. Since the model is intended to simulate the performance of an extended train of identical microbunches, the axial space charge debunching forces should be zero by symmetry at the midplane between microbunches. Thus, we make the additional adjustment of removing the linear portion of Eq. (A17) through the range between $\psi_{b}-\pi<\psi<\psi_{b}+\pi$.

The Lorentz force on each electron due to an axial $E$ field is

$$
\frac{d \gamma}{d z}=-\frac{e E_{z}}{m c^{2}}
$$

Combining the previous equations, we obtain 


$$
\begin{array}{r}
\frac{d \gamma}{d z}=F_{2 \mathrm{D}} \sqrt{\frac{\pi}{2}} \frac{e^{2} n_{e o} f_{b}}{m c^{2} \varepsilon_{0}} \frac{\sigma_{b}}{k_{s}}\left\{\operatorname{erf}\left[\left(\psi-\psi_{b}\right) / \sqrt{2} \sigma_{b}\right]-\operatorname{erf}\left(\pi / \sqrt{2} \sigma_{b}\right)\left(\frac{\psi-\psi_{b}}{\pi}\right)\right\} \\
\psi_{b}-\pi<\psi<\psi_{b}+\pi
\end{array}
$$

This longitudinal space charge correction is used to simulate the additional debunching forces due to space charge within the drift region. Note that the debunching forces tend to reduce the energy modulation induced in the buncher, thus retarding the bunching process. The space charge interaction is largely elastic, so that a modulated energy distribution similar to the original distribution is recovered if the beam is allowed to continue drifting to the point where it becomes unbunched once again.

In the present space charge model, three-dimensional effects are included only through the simplistic 2D correction factor. An extensive effort to improve the space charge model has not been warranted since future experiments are moving to higher energies and shorter bunching sections where space charge becomes negligible.

Further developments of the STELLA simulation model are expected to concentrate on modeling new stagedacceleration configurations (e.g., Fig. 9) that take advantage of the higher laser intensities becoming available. For example, the IFEL buncher portion of the simulation shown in Fig. 10 utilizes a revised FEL model suitable for an ultrashort undulator with a $B$-field distribution dominated by end field effects. In that case, the wiggleaveraged equations [(A2) and (A3)], which are based on interaction with a quasisinusoidal undulator magnetic field, are not applicable. Hence, nonwiggle-averaged equations are used instead.

[1] J. People, Jr., in Proceedings of the 1997 Particle Accelerator Conference, Vancouver, Canada, edited by M. Comyn, M. K. Craddock, M. Reiser, and J. Thomson (IEEE, Piscataway, NJ, 1998), p. 29.

[2] G. B. Coutrakon, J. M. Slater, and A. Ghebremedhin, in Proceedings of the 1999 Particle Accelerator Conference, New York, edited by A. Luccio and W. MacKay (IEEE, Piscataway, NJ, 2000), p. 11.

[3] H. Winick, in Proceedings of the 1997 Particle Accelerator Conference, Vancouver, Canada (Ref. [1]), p. 37.

[4] See, for example, Nature Structural Biology, Synchrotron Supplement, edited by S. M. Mini, S. R. Stock, D. L. Perry, and L. J. Terminello (Materials Research Society, Warrendale, PA, 1998); Proceedings of the Symposium on Applications of Synchrotron Radiation Techniques to Material Science, edited by S. M. Mini, S. R. Stock, D. L. Perry, and L. J. Terminello (Materials Research Society, Warrendale, PA, 1998)

[5] M. J. Kelley, H. F. Dylla, G. R. Neil, L. J. Brillson, D. P. Henkel, and H. Helvajian, Proc. SPIE Int. Soc. Opt. Eng. 2703, 15 (1996).
[6] Y. Jongen, in Proceedings of the 1997 Particle Accelerator Conference, Vancouver, Canada (Ref. [1]), p. 3770.

[7] W.P. Leemans and E. Esarey, in Advanced Accelerator Concepts: Eighth Workshop, edited by W. Lawson, C. Bellamy, and D. Brosius, AIP Conf. Proc. No. 472 (AIP, New York, 1999), p. 174.

[8] W. D. Kimura, A. van Steenbergen, M. Babzien, I. Ben-Zvi, L. P. Campbell, C. E. Dilley, D. B. Cline, J. C. Gallardo, S. C. Gottschalk, P. He, K. P. Kusche, Y. Liu, R. H. Pantell, I. V. Pogorelsky, D. C. Quimby, J. Skaritka, L. C. Steinhauer, and V. Yakimenko, Phys. Rev. Lett. 86, 4041 (2001).

[9] Y. Liu, X. J. Wang, D. B. Cline, M. Babzien, J. M. Fang, J. Gallardo, K. Kusche, I. Pogorlesky, J. Skaritka, and A. van Steenbergen, Phys. Rev. Lett. 80, 4418 (1998).

[10] W. D. Kimura, M. Babzien, I. Ben-Zvi, L. P. Campbell, D. B. Cline, R. B. Fiorito, J. C. Gallardo, S. C. Gottschalk, P. He, K. P. Kusche, Y. Liu, R. H. Pantell, I. V. Pogorelsky, D. C. Quimby, K. E. Robinson, D. W. Rule, J. Sandweiss, J. Skaritka, A. van Steenbergen, L.C. Steinhauer, and V. Yakimenko, in Advanced Accelerator Concepts: Eight Workshop (Ref. [7]), p. 563.

[11] K. P. Kusche, M. Babzien, I. Ben-Zvi, L. P. Campbell, D. B. Cline, R. B. Fiorito, J. C. Gallardo, S. C. Gottschalk, P. He, W. D. Kimura, Y. Liu, R. H. Pantell, I. V. Pogorelsky, D. C. Quimby, K. E. Robinson, D. W. Rule, J. Sandweiss, J. Skaritka, A. van Steenbergen, L. C. Steinhauer, and V. Yakimenko, in Advanced Accelerator Concepts: Eight Workshop (Ref. [7]), p. 573.

[12] R. B. Palmer, J. Appl. Phys. 43, 3014 (1972).

[13] T.C. Marshall, Free-Electron Lasers (MacMillan, New York, 1985), pp. 24-26.

[14] I. V. Pogorelsky, J. Fischer, K. P. Kusche, M. Babzien, N. A. Kurnit, I. J. Bigio, R. F. Harrison, and T. Shimada, IEEE J. Quantum Electron. 31, 556 (1995).

[15] L. C. Steinhauer and W. D. Kimura, Phys. Rev. ST Accel. Beams 2, 081301 (1999).

[16] I. V. Pogorelsky, I. Meshkovsky, A. Dublov, I. Pavlishin, Yu. A. Boloshin, G. B. Deineko, and A. Tsunemi, in Proceedings of LASERS'98, Tucson, AZ, 1998, edited by V. J. Cocoran and T. A. Goldman (STS Press, McLean, VA, 1999), p. 911.

[17] N. M. Kroll, P. L. Morton, and M. N. Rosenbluth, IEEE J. Quantum Electron. 17, 1436 (1981).

[18] J. Struckmeier, J. Klabunde, and M. Reiser, Part. Accel. 15, 47 (1984).

[19] J. Murphy, Brookhaven National Laboratory Report No. BNL-42333, Version 3.0, 1993, p. 5.

[20] See, for example, D. C. Quimby, Proc. SPIE Int. Soc. Opt. Eng. 738, 103 (1987).

[21] A.E. Siegman, Lasers (University Science Books, Mill Valley, CA, 1986), p. 664.

[22] C. A. Brau, Free Electron Lasers (Academic, Boston, 1990), p. 151. 